\title{
Electrostimulators for Acupuncture: Safety Issues
}

\author{
C. DAVID LYTLE, Ph.D., ${ }^{1}$ BRENTON M. THOMAS, ${ }^{2}$ EDWARD A. GORDON, ${ }^{1}$ \\ and VICTOR KRAUTHAMER ${ }^{1}$
}

\begin{abstract}
Three representative electrostimulators were evaluated to determine whether they meet the manufacturers' labeled nominal output parameters and how the measured parameters compare with a safety standard written for implanted peripheral nerve stimulators. The pulsed outputs (pulse width, frequency, and voltage) of three devices were measured with an oscilloscope across a 500-ohm resistance, meant to simulate subdermal tissue stimulated during electroacupuncture. For each device, at least two measured parameters were not within $25 \%$ of the manufacturer's claimed values. The measured values were compared with the American National Standard ANSI/AAMI NS15 safety standard for implantable peripheral nerve stimulators. Although for two stimulators the pulse voltage at maximum intensity was above that specified by the standard, short-term clinical use may still be safe because the standard was written for long-term stimulation. Similarly, the net unbalanced DC current, which could lead to tissue damage, electrolysis, and electrolytic degradation of the acupuncture needle, was within the limits of the standard at 30 pulses per second, but not at higher frequencies. The primary conclusions are (1) that the outputs of electrostimulators must be calibrated and (2) that practitioners must be adequately trained to use these electrostimulators safely.
\end{abstract}

\section{INTRODUCTION}

$\mathbf{T}$ he use of electricity to stimulate the sites punctured by acupuncture needles became common in China in the late 1950s (White, 1998) and has been investigated in the United States since at least the early 1970s. Their purpose is to provide sustained stimulation to an acupuncture point that is controlled with regard to stimulation intensity and frequency and is equivalent or superior to, and in place of, manual twirling of the needle (Lee and Cheung, 1978). Electroacupuncture is accomplished by connecting a low-amperage electrical pulse generator to a pair of inserted acupuncture needles via small "alligator" clips (or to a single needle, plus a hand-held electrode to complete the electrical circuit).

Based on adverse event surveys in Norway (Norheim and Fønnebø, 1996), Australia (Bensoussan and Myers, 1996) and Japan (Yamashita et al., 1999) and numerous additional case reports of injuries in the published literature (Peacher, 1975; Lao, 1996; Rosted, 1996), the practice of acupuncture has been found to have very low adverse event rates (NIH, 1998), with nearly all such events arising from invasive use of the acupuncture needle (Lao, 1996; Rosted, 1996; MacPherson, 1999). However, injuries from electrostimulation could be subtle or delayed, and therefore, perhaps not reported (Omura, 1987). The purpose of this study was

\footnotetext{
${ }^{1}$ Office of Science and Technology, FDA Center for Devices and Radiological Health, Rockville, Maryland.

${ }^{2}$ Department of Biomedical Engineering, Marquette University, Milwaukee, Wisconsin.
} 
to determine whether there is the potential to do significant harm through electric shock, excessive current, or other means. This was approached by determining the output of a few representative electrostimulators and comparing the results with the most relevant safety standard, taking into account how the stimulators are used in therapeutic practice. Although most electrostimulators provide electrodes for external, transcutaneous stimulation (transelectrical nerve stimulation [TENS]-like), this study was limited to electrical stimulation as intended to be applied directly to the needles. It consisted of measurements of the electric current from the pulse generators and did not consider possible effects that could occur at the electrode-tissue interface as a result of electrochemical processes at the electrode.

\section{MATERIALS AND METHODS}

\section{Electrostimulators}

Three stimulators were obtained for evaluation. They were chosen to be representative of those investigated in the United States and included selections of differing specifications, size, cost, manufacturer, and country of origin. The listed specifications of each stimulator are shown in Table 1. The specific brands are not identified because only one specimen of each type was investigated and the specimen-tospecimen variation was not determined. Two of the stimulators had an output voltage switch with a low position for use with needle acupuncture and a high position for transcutaneous stimulation. The measurements reported here were limited to the position relevant to needle acupuncture.

All stimulators had pulsed output with variable frequencies. Two devices (Brands B and C) had dual-frequency controls allowing outputs that were continuous, intermittent, or of mixed pulse rates, i.e., consisting of alternating periods at one pulse rate with periods at a different pulse rate. The two frequency controls were independent of each other. The designs of the stimulators were not investigated, nor were possible failure modes. Only their outputs at selected settings were measured.

\section{Relevant safety standard}

Although some aspects of acupuncture therapy cannot be explained by a neurostimulation model (Birch and Felt, 1999), safety considerations for electrically stimulated acupuncture needles suggest assuming that model anyway because nerve tissue is particularly sensitive to electrical current injury. The safety standard developed for implantable peripheral nerve stimulators (ANSI, 1995) is, in our opinion, the most appropriate. It includes considerations for electrical current injury specifically to peripheral nerves and to tissue in general, as well. American National Standard ANSI/AAMI NS15-1995 (NS15) was developed by the Implantable Neurostimulator Subcommittee of the Association for the Advancement of Medical Instrumentation (AAMI) Neurosurgery Committee and approved in 1995 by the Amer-

Table 1. Stated Parameters of Pulsed Electrostimulators Used in Acupuncture ${ }^{a}$

\begin{tabular}{|c|c|c|c|c|}
\hline Parameter & Brand $A$ & Brand $B$ & Brand C & Typical values ${ }^{b}$ \\
\hline Pulse frequency $(\mathrm{Hz})$ & $0.5-500$ & $\begin{array}{l}1-30 \text { or } \\
10-300\end{array}$ & $\begin{array}{c}0-100 \text { or } \\
0-1000\end{array}$ & $3-100$ \\
\hline Pulse width $(\mu s)$ & 70 & 150 or $50^{c}$ & 400 or $40^{c}$ & $50-500$ \\
\hline Max voltage (V) & 10 & 16 & 4 & $\leq 20$ \\
\hline Max current (mA) & 20 & 32 & 8 & $10-50$ \\
\hline Waveform polarity ${ }^{d}$ & Same & Alternating & Same & Same \\
\hline Origin & Japan & Japan & China & \\
\hline Approximate cost & $\$ 100-200$ & $>\$ 400$ & $\$ 100-200$ & \\
\hline
\end{tabular}

aA 500 -ohm test load was used to determine the values.

bFrom: White, 1998.

cLonger pulse width with the low pulse frequency setting, short with the high setting.

dBasic waveform is asymmetric biphasic square, e.g., a positive square wave followed by a negative phase that decreases rapidly (see Fig. 1). Each successive pulse may have the same polarity or may alternate in polarity. 
Table 2. Safety Parameters for Peripheral. Nerve ELECTROSTIMULATORS ${ }^{\mathrm{a}}$

\begin{tabular}{lcc}
\hline Parameter & NS15 & Preferred $^{\mathrm{b}}$ \\
\hline Pulse frequency $(\mathrm{Hz})$ & $1-1,500$ & $1-300$ \\
Pulse width $(\mu \mathrm{s})$ & $1-2,000$ & $1-1,000$ \\
Max voltage $(\mathrm{V})$ & 15 & 15 \\
Max current $(\mathrm{mA})$ & 30 & 9 \\
Waveform & Balanced & Balanced \\
\hline
\end{tabular}

aA 500 -ohm test load should be used to determine these values.

bModified values derived from physiologic considerations and preferred by the authors; see text for rationale.

'Net DC current should be less than $10 \mu \mathrm{A}$.

ican National Standard Institute, Inc. The standard established minimum labeling, safety, and performance requirements for stimulators that contact peripheral nerves directly. As part of the performance requirements, the stimulation parameters specified by the standard for safe and effective current to stimulate peripheral nerves were established and are presented in Table 2. In addition, the waveform of the current pulses should be balanced to prevent the net DC current through the electrodes from exceeding $10 \mu \mathrm{A}$. Net dc current can damage any tissue and is not specific to nerves (Omura, 1987).

Additional considerations suggest that the stimulation parameters should be restricted beyond those provided by this safety standard. These additional restrictions are based on known physiologic properties of neurons. For nerve stimulation, the pulse frequency need not exceed 300 pulses per second because the peripheral nerves typically cannot follow faster frequencies (Talbot et al., 1968). Similarly, the pulse width should not exceed $1000 \mu$ s (the maximum chronaxie in human nerve [Reilly, 1998]), because nothing would be gained from a longer pulse, and many electrodes cannot carry long currents. Because an average output current exceeding $10 \mathrm{~mA}$ can heat surrounding tissues (ANSI, 1986), the average output voltage should be limited to 5 volts. Thus, to avoid heating with the above pulse limits $(300 \mathrm{~Hz}, 1-$ ms pulse width), the maximum voltage of each pulse should not exceed 16 volts, similar to the NS15 limit. These values are also shown in Table 2.

\section{Measurements of stimulator output}

Three parameters were measured: pulse frequency, waveform, and voltage during the pulse. Frequency measurements for each device were determined with a Philips PM 3350 $50 \mathrm{Mhz}$ Oscilloscope (Fluke International Corp., Everett, WA) connected in parallel to a 500 -ohm resistor, as specified by NS15. The other parameters were recorded with a Fluke $9750 \mathrm{MHz}$ Scopemeter (Fluke International Corp.), also with a 500-ohm resistor. In one test several different resistors (100-1000 ohm) were used to determine whether each pulse generator acted primarily as a voltage source, or as a current source. The accuracy of the pulse frequency settings were checked by recording actual pulse frequencies directly from the oscil-

Table 3. Measured Pulse Frequencies ${ }^{a, b}$

\begin{tabular}{lccc}
\hline & Brand A & Brand B & Brand C \\
\hline $\begin{array}{l}\text { Nominal pulse } \\
\text { frequency (Hz) }\end{array}$ & & & \\
$2-2.5$ & $1.9 / 2.5$ & $3.5-4.0 / 2$ & $\mathrm{NPc}$ \\
5.5 & $4.2-4.3 / 5.5$ & & $\mathrm{NP}$ \\
10 & $17.0-18.1 / 20$ & $28.0 / 2 \times 10$ & $20.7-21.1 / 20$ \\
20 & $24.3 / 30$ & $31.4-32.0 / 30$ & $30 / 2443-45$ \\
$24-30$ & $59.6 / 4.5 \times 10$ & $65.7-71.9 / 45$ & \\
$35.4 / 43$ & $64.5 / 80$ & & $98-100 / 70$ \\
$70-80$ & & $119-139 / 10 \times 10$ & $100-102 / 100$ \\
100 & &
\end{tabular}

aMeasured value compared to nominal value, presented as "measured/nominal." Nominal value when high range was used is indicated by " $\times 10$," e.g., $2 \times 10=20 \mathrm{~Hz}$ using the high range.

b500-ohm test load was used to determine this value.

'NP, no pulse generated at frequency settings below $10 \mathrm{~Hz}$. 
loscope. The pulse voltages were measured directly from the oscilloscope, as the average of the height of the square wave portion of the pulse (NS15). To record the pulse waveforms, digitized data were taken directly from the oscilloscope.

\section{RESULTS}

\section{Pulse frequency}

Although the formal manner of expressing pulse frequency is in pulses per second (pps), we will use Hertz $(\mathrm{Hz}$, or cycles per second) to conform with that found on the instruments and in their accompanying literature. The measured pulse frequencies were compared with the nominal frequency settings (Table 3 ). The instruments did not allow precise control over the frequency, i.e., setting the pulse frequency was only reproducible to within $\pm 5 \%$. The relationship between measured frequency values and nominal values varied from one brand of electrostimulator to another and from one frequency range to another. The ratio of the measured frequency to the nominal frequency provided a "correction factor" that can be used to discuss the accuracy of the frequency settings (Table 4). In general, the nominal frequency settings were not accurate. Fewer than half the measured values were within $25 \%$ of the nominal values. One electrostimulator, brand $A$, had all measured values lower than the nominal values, but within $25 \%$; the other brands had at least one measured value $50 \%$ higher than the nominal value. The greatest inaccuracies tended to be in the low frequency range $(2-10 \mathrm{~Hz})$.

TABle 4. FACTORS FOR CORRECTING NOMINAL FREQUENCIES

\begin{tabular}{lccc}
\hline & Brand A & Brand B & Brand C \\
\hline $\begin{array}{l}\text { Pulse frequency } \\
\text { Range (Hz) }\end{array}$ & & & \\
$2-10$ & $0.76-0.77$ & $1.66-1.88$ & $\mathrm{NP}$ \\
$20-45$ & $0.81-0.88$ & $1.06-1.40$ & $1.05-1.53$ \\
$70-100$ & 0.81 & 1.29 & $1.00-1.41$ \\
\hline
\end{tabular}

acalculated from values in Table 3 , equal to the measured frequency divided by nominal frequency.

\section{Waveform}

The pulse waveforms are shown in Figure 1. In general, the waveforms were similar for the different devices, asymmetric biphasic square waves, i.e., a positive square wave followed by reversed polarity and an asymptotic recovery period that is reminiscent of the voltage from a discharging capacitor. The waveform of Brand B was different in having the individual pulses alternating in polarity, with one pulse having a positive square wave with a small negative recovery and the next pulse having a negative square wave with a small positive recovery, etc.

The extent of balance in the waveforms was determined for those devices that had unidirectional pulses (Brands $\mathrm{A}$ and $\mathrm{C}$ ). The pulses of Brand $\mathrm{A}$ had $93.0 \%$ recovery during the asymptotic recovery period, resulting in a net current at $30 \mathrm{~Hz}$ and maximum intensity of 7.4 $\mu \mathrm{A}$ (calculated using 500-ohm resistance). Brand $C$ had a $91.7 \%$ recovery and a net current at $30 \mathrm{~Hz}$ and maximum intensity of $24 \mu \mathrm{A}$. Because Brand B had identical pulses of alternating polarity, its net current was zero, i.e., balanced.

\section{Pulse width}

The pulse widths were reproducible across different frequencies, except, of course, in instances where the pulse width changed with pulse frequency range (Brands $B$ and $C$ ). They were also relatively close $( \pm 17 \%)$ to the quoted nominal values (Table 5), except for Brand A where they were $50 \%$ to $60 \%$ above the quoted value. The measured widths were within the range of the safety parameters (compare values with those in Table 2).

\section{Voltage}

The voltages were measured from pulses of nominal 10 and $30 \mathrm{~Hz}$ produced at maximum intensity for the settings used for electrostimulation of acupuncture needles. Again, the measured values were different from the quoted nominal values (Table 5). Whereas Brand B had a voltage less than half the nominal value, two devices (Brands A and C) had significantly higher voltages. Furthermore, the latter two de- 
$\mathbf{A}$

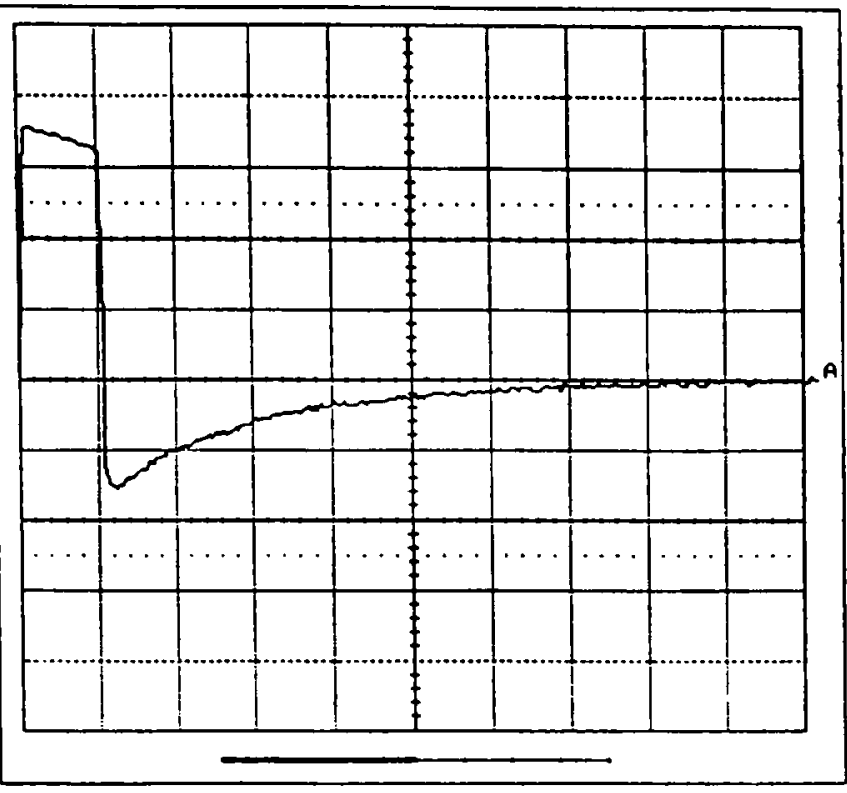

$\mathbf{B}$

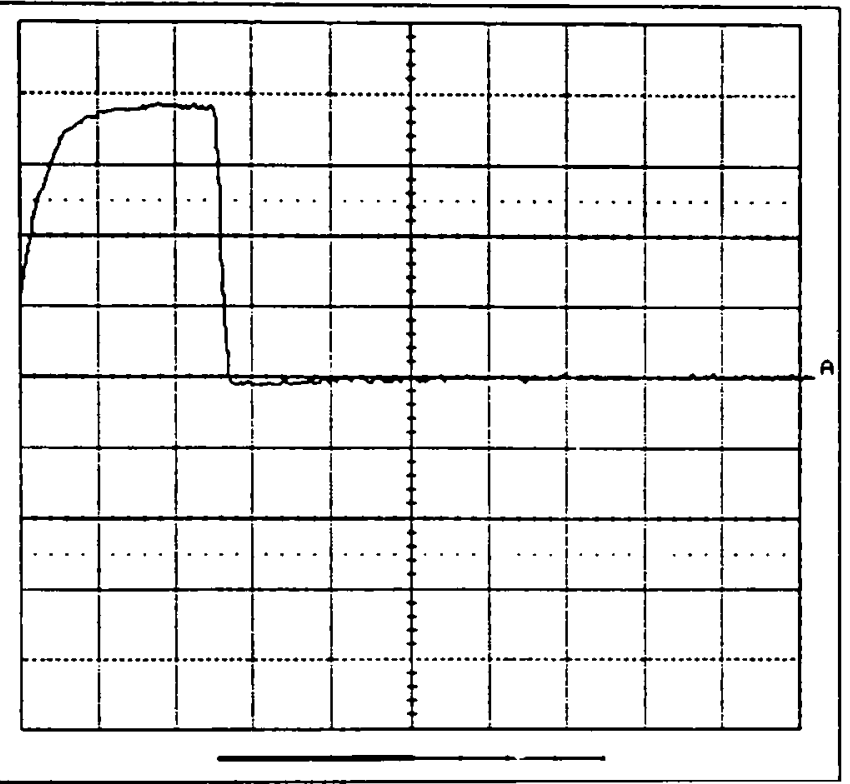

C

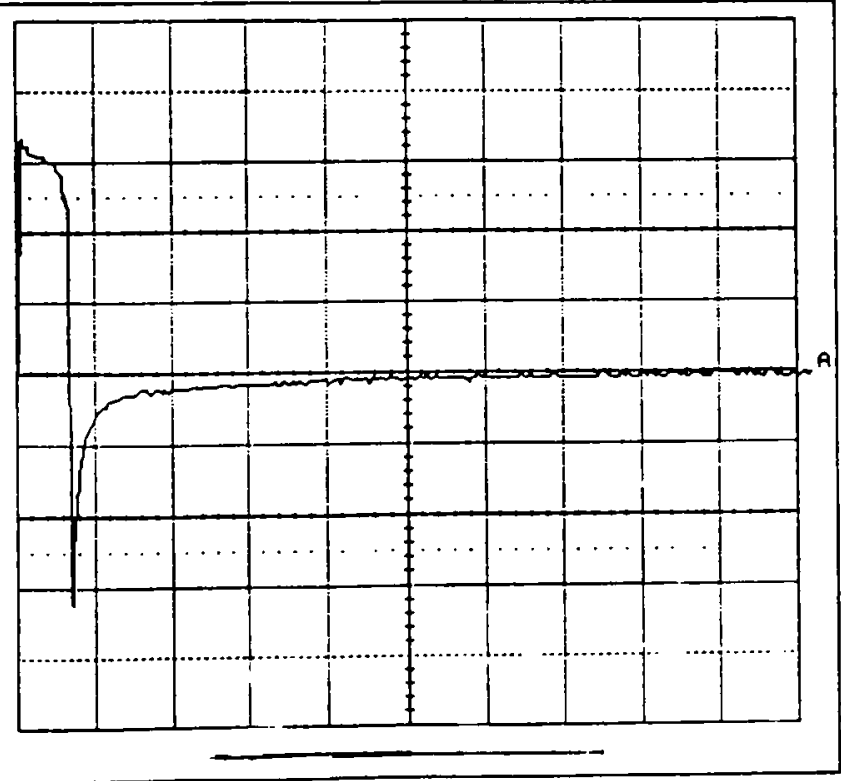

FIG. 1. Typical waveforms of pulses from the electrostimulators. Axes were adjusted to accommodate waveform: (A) brand A (divisions $=5 \mathrm{~V}, 100 \mu \mathrm{s}$ ); (B) brand B $(2 \mathrm{~V}, 20 \mu \mathrm{s})$; (C) brand C (5 V, $500 \mu \mathrm{s})$.
Table 5. Measured Stimulator Values ${ }^{a, b}$

\begin{tabular}{lccc}
\hline Parameter & Brand A & Brand B & Brand C \\
\hline $\begin{array}{l}\text { Pulse width } \\
\text { ( } \mu \text { s) }\end{array}$ & $104-111 / 70$ & $50-54 / 50$ & \\
& & $147-150 / 150$ & $326-336 / 400$ \\
$\begin{array}{l}\text { Max voltage } \\
(V)\end{array}$ & $17.0-17.5 / 10$ & $6.8-7.6 / 16$ & $15.1-15.7 / 4$ \\
& & &
\end{tabular}

a500-ohm test load was used to determine these values.

bMeasured value compared to manufacturer's nominal value (measured/nominal).

'Determined for $10 \mathrm{~Hz}$ and $30 \mathrm{~Hz}$ pulses at maximum intensity.

vices at maximum intensity exceeded the NS15 maximum voltage limit. All three pulse generators changed in output voltage when resistance was changed. Brands B and C acted primarily as current sources (i.e., the output voltage increased by more than $50 \%$ for a $100 \%$ increase in resistance), and Brand $A$ acted primarily as a voltage source (i.e., less than a $50 \%$ increase in voltage for a $100 \%$ increase in resistance).

\section{DISCUSSION}

Three aspects of these results require elaboration: the precision of the stimulators' outputs and their ramifications, the inherent safety (or danger) of the stimulator, and the safety in the hands of a qualified practitioner. In each case, the results are important to both the regulators of such devices and the practitioners who use them.

\section{Lack of precise pulsed output of stimulators} for electroacupuncture

The most bothersome aspect of the poor precision of the stimulator outputs is recognition that most of the data in the acupuncture literature were developed from experiments that employed similar devices. Few, if any, investigators have actually measured the output of their stimulators, instead simply accepting the claims of the device manufacturer(s). In one sense, some uncertainty in the pulse frequency may not be serious. For example, the evidence used to support a frequency dependence in the antinociceptive mechanisms of electro- 
acupuncture differentiate only between a broad range of low frequencies $(1-15 \mathrm{~Hz})$ and a broad range of higher frequencies (45-100 $\mathrm{Hz}$ ) (Han et al., 1984; Han, 1987). On the other hand, accurate knowledge of the voltage and the resultant unbalanced current will be important for safe clinical use and for adequate interpretation of electroacupuncture experiments. Thus, better characterization of the experimental devices is required in the future, perhaps by following the guidelines used in this study.

\section{Implications for safe use}

While the pulse frequencies and pulse widths of these devices were within the limits specified by a nerve stimulator safety standard, the maximum pulse voltage and the unbalanced current are of concern. Both excessive electrical current and unbalanced, net current can cause neural damage (Shannon, 1992) and damage to other tissue. This is particularly true with the thin acupuncture needles. As the needle surface area decreases the maximum charge density increases, and charge and charge density act as cofactors in determining neural damage (McCreery et al., 1990; Shannon, 1992). Higher charge densities are more likely to cause electrochemical reactions at the electrode. Therefore, as the needle diameter decreases, the possibility for local damage increases for a given amount of current. Moreover, because two of the tested stimulators acted primarily as current sources, charge density could be increased if current were applied as a needle is withdrawn because of the decrease in needle surface area exposed to tissue. Therefore, the current should always be turned off before the needle is withdrawn.

The extent of unbalanced, net current depends on the "intensity" and on the pulse frequency. The net current will be directly proportional to the voltage (at a fixed resistance) and also to the frequency. Thus, in order to maintain a safe level of net current, a change in intensity requires a reciprocal change in frequency. For example, the Brand A stimulator produced a net current of $7.6 \mu \mathrm{A}$ at maximum intensity and $30 \mathrm{~Hz}$. To avoid exceeding $10 \mu \mathrm{A}$ at maximum intensity, the maximum safe fre- quency would be $39.5 \mathrm{~Hz}(30 \times 10 / 7.6)$. Similarly, if $100 \mathrm{~Hz}$ were used, an intensity of only $39.5 \%$ of maximum would avoid exceeding the $10 \mu \mathrm{A}$ limit. Note that to avoid charge imbalance it becomes important to know the exact pulse frequency.

While use of the electrostimulators at maximum intensity may provide excessive voltage, in standard practice the maximum intensity is not used. In actual practice, the acupuncturist is likely to increase the intensity until there is slight muscle twitching or other indication by the patient (e.g., tingling or even pain) and then decrease the intensity slightly (White, 1998). While the maximum intensity is not used normally, the actual voltage used is not standardized. Ten volts (which produce about $20 \mathrm{~mA}$ in muscle tissue) is adequate to depolarize nerve endings (White, 1998). Published studies of electroacupuncture on humans include low values of 1-3 V (Voll, 1975; Han et al., 1984) and high values of 9-12 V (Han, 1987; Christiensen et al., 1989; Deluze et al., 1992). It is likely that these quoted values were not measured but were deduced from the settings on their individual devices combined with manufacturers information. Our data (Table 5) indicate the unreliability of such deductions. However, it is plausible that the actual voltages in standard practice are usually less than $15 \mathrm{~V}$.

\section{Other safety issues for electroacupuncture}

Thus, normal use of these stimulators probably does not exceed the NS15 safety standard, depending on the intensity and frequency selections. On the other hand, the standard may be exceeded when there is inadequate information about the stimulator and/or the above issues are not considered. Care must also be taken to avoid extreme situations, such as using the high-intensity range meant only for transdermal stimulation. Often harmful levels of stimulation cause pain and patient discomfort, which, of course, should be monitored.

In addition, there are other hazards of electrostimulation. Because of the electrical nature of control of the heart and respiration, stimulation near the carotid sinus or the heart of cardiac patients, especially those with demandtype cardiac pacemakers, must be avoided 
(Fujiwara et al., 1980; Omura, 1987). Similarly, situations should be avoided during which electric current travels across the heart. Stimulation near the pregnant uterus is in the same category (White, 1998). Needles can be broken or damaged during violent muscle contraction caused by excessive stimulation (White, 1998). Electrolysis near the needle surface and electrolytic degradation of the needle can occur if there is excessive unbalanced electric current, occasionally resulting in local injury (White, 1998). That is why the devices have some mechanism to balance the current, i.e., by having a recovery period with reversed current for each pulse or by reversing the polarity of alternating pulses.

For the most part, with present standards of practice these problems are "rarely encountered" (Reichmanis, 1988) and electrostimulators are capable of safe application. However, the electrostimulators are also capable of being used in manners that are not safe, particularly at higher pulse frequencies. The primary injury would result from excess current and would be local to the electrified needle(s).

Care must be used with electrostimulated needle acupuncture. Training of the user is critical and must be adequate, particularly regarding use of high frequencies. Electronic characterization and calibration of the stimulator is needed in order to know the actual output parameters in clinical use. Thus, practitioners who investigate electrostimulators should have their instruments calibrated. Furthermore, any future research with electroacupuncture must use well-characterized stimulators.

While this study may not be satisfying with regard to identifying the "best" electrostimulator, it points the way to what parameters are important: balanced output to eliminate, or at least minimize, net DC current; accurate voltage and frequency indicators; and minimal variation among instruments. In addition, there should be no leakage current between electrodes in multichannel devices (Omura, 1987).

\section{ACKNOWLEDGMENT}

The authors gratefully acknowledge Tom $\mathrm{Ri}$ ihimaki, OMS Medical Supplies Inc., Braintree,
Massachusetts, for helpful discussions and loan of two of the electrostimulators investigated in this study and Brian Beard, FDA, for conversion of digitized waveforms onto spreadsheets for analysis of unbalanced current.

\section{AUTHORS' NOTE}

This study was intended merely to identify the safety issues that occur in the investigation of electroacupuncture and is not intended to imply endorsement of electroacupuncture by the Food and Drug Administration. At this time, electroacupuncture is not an approved use of the electrostimulators tested.

\section{REFERENCES}

ANSI. Trancutaneous Electrical Nerve Stimulators. NS4. American National Standard Institute, Inc., Arlington, 1986.

ANSI. Implantable Peripheral Nerve Stimulators. NS15. American National Standard Institute, Inc., Arlington, 1995.

Bensoussan A, Myers SP. Towards a safer choice. The practice of traditional Chinese medicine in Australia. New South Wales, Australia: Faculty of Health, University of Western Sydney, Macarthur, 1996.

Birch SJ, Felt RL. Understanding Acupuncture. Edinburgh: Churchill Livingstone, 1999: Chapter 3.

Christensen PA, Noreng M, Andersen PE, Nielsen JW. Electroacupuncture and postoperative pain. $\mathrm{Br} J$ Anaesth 1989;62:258-262.

Deluze C, Bosia L, Zirbs A, Chantraine A, Vischer TL. Electroacupuncture in fibromyalgia: Results of a controlled trial. Br Med J 1992;305:1249-1252.

Fujiwara H, Taniguchi K, Takeuchi J, Ikezono E. The influence of low frequency acupuncture on a demand pacemaker. Chest 1980;78:96-97.

Han JS. Central neurotransmitters and acupuncture analgesia. In: Pomeranz B, Stux G, eds. Scientific Basis of Acupuncture. Springer-Verlag, 1989:7-33.

Han JS, Xie GX, Ding XZ, Fan SG. High and low frequency electroacupuncture analgesia are mediated by different opioid peptides. Pain Suppl 1984;2:543.

Lao LX. Safety issues in acupuncture. J Altern Complement Med 1996;2:27-31.

Lee JF, Cheung CS. Current Acupuncture Therapy. Hong Kong: Medical Book Publications, 1978:41-48.

MacPherson $\mathrm{H}$. Fatal and adverse events from acupuncture: Allegation, evidence, and the implications. J Altern Complement Med 1999;5:47-56.

McCreery DB, Agnew WF, Yuen TGH, Bullara L. Charge density and charge per phase as cofactors in neural in- 
jury induced by electrical stimulation. IEEE Trans Biomed Eng 1990;37:996-1001.

NIH. NIH Consensus Conference. Acupuncture. JAMA 1998;280:1518-1524.

Norheim AJ, Fønnebø V. Acupuncture adverse effects are more than occasional case reports: results from questionnaires among 1135 randomly selected doctors, and 197 acupuncturists. Complement Ther Med 1996;4: 8-13.

Omura Y. Basic electrical parameters for safe and effective electro-therapeutics [electro-acupuncture, TES, TENMS (or TEMS), TENS and electro-magnetic field stimulation with or without drug field] for pain, neuromuscular skeletal problems, and circulatory disturbances. Acupunct Electrother Res Int J 1987;12:201-225.

Peacher WG. Adverse reactions, contraindications and complications of acupuncture and moxibustion. Am J Chin Med 1975;3:35-46.

Reichmanis M. Electroacupuncture. In: Marino AA, ed. Modern Bioelectricity. New York: Marcel Dekker, Inc., 1988:757-782.

Reilly JP. Applied Bioelectricity. New York: Springer-Verlag, 1998:244-248.

Rosted P. Literature survey of reported adverse effects associated with acupuncture treatment. Am J Acupunct 1996;24:27-34.
Shannon RV. A model of safe levels for electrical stimulation. IEEE Trans Biomed Eng 1992;39:424-426.

Talbot WH, Darian-Smith I, Kornhuber HH, Moutcastle VB. The sense of flutter vibration: Comparison of the human capacity with response patterns of mechanoreceptive afferents from the monkey hand. J Neurophysiol 1968;31:301-334.

Voll R. Twenty years of electroacupuncture therapy using low-frequency current pulses. Am J Acupunct 1975;3:291-314.

White A. Electroacupuncture and acupuncture analgesia. In: Filshie J, White A, eds. Medical Acupuncture. Edinburgh: Churchill Livingstone, 1998:153-175.

Yamashita H, Tsukayama H, Tanno Y, Nishijo K. Adverse events in acupuncture and moxibustion treatment. A six-year survey at a national clinic in Japan. J Altern Complement Med 1999;5:229-236.

Address reprint requests to:

C. David Lytle, Ph.D.

P.O. Box 396

Arroyo Seco NM 87514-0396 